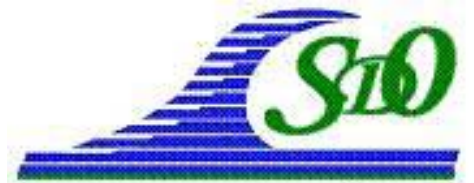

XI ${ }^{\text {èmes }}$ Journées Nationales Génie Côtier - Génie Civil

Les Sables d'Olonne, 22-25 juin 2010

DOI:10.5150/jngcgc.2010.083-K @ Editions Paralia CFL

disponible en ligne - http://www.paralia.fr-available online

\title{
Simulation de la stabilité des digues en enrochement par la méthode d'Analyse de Déformations Discontinues (DDA)
}

\author{
Sami KAIDI ${ }^{1}$, Abdellatif OUAHSINE ${ }^{1}$, Mohamed ROUAINIA ${ }^{2}$, \\ François HISSEL $^{3}$, Philippe SERGENT ${ }^{3}$
}

1. Université de Technologie de Compiègne (UTC), Centre de Recherches de Royallieu, Laboratoire Roberval UMR CNRS 6253, 60205 Compiègne, France.

sami.kaidi@utc.fr,ouahsine@utc.fr

2. School of Civil Engineering and Geosciences, University of Newcastle, NE1 7RU,

Newcastle, UK.

m.rouainia@newcastle.ac.uk

3. Centre d'Etudes Techniques Maritimes et Fluviales (CETMEF),

2 Boulevard Gambetta, 60321 Compiègne, France.

Francois.Hissel@developpement-durable.gouv.fr

Philippe.Sergent@developpement-durable.gouv.fr

\section{Résumé :}

La protection des côtes contre les effets érosifs de la houle est assurée très souvent par la construction de digues en enrochements. De nombreux travaux ont été réalisés par le passé pour estimer la stabilité de ces digues en utilisant des modèles simples issus d' expériences in situ ou de modèles réduits tels que les modèles de Hudson ou de Van der Meer. Cependant, ces modèles ne tiennent compte ni de la taille et de la forme des blocs, ni de leur juxtaposition.

Dans ce travail, on se propose d'étudier numériquement la stabilité de ces structures, en tenant compte de la forme des blocs, de leurs dispositions, des forces de contact entre blocs, des caractéristiques hydrodynamiques du fluide, et des effets rétroactifs de l'écoulement du fluide sur les blocs. Il s'agit d'un couplage fluide-structure où la structure est supposée formée d'éléments discrets (blocs) susceptibles de se déformer. Les mouvements des structures sont alors modélisés par un modèle numérique d'éléments discrets (DEM) basé sur la méthode de l'Analyse de Déformations Discontinues (DDA), et adoptant l'algorithme du "Lagrangien Augmenté" pour la prise en compte des liaisons des contacts. Les résultats du modèle sont validés par des expérimentations sur des structures simples en canal à courant.

Mots clés :

Couplage fluide-structure - Eléments discrets (DEM) - Eléments finis (FEM) Méthode DDA - Digues - Enrochements - Contact - Simulation numérique - Stabilité de la digue 


\section{Introduction}

Le présent travail concerne l'étude de la stabilité des digues à talus en enrochements naturels ou artificiels (figure 1), soumises aux sollicitations naturelles (la houle, les courants, le réchauffement climatique ...). Plusieurs lois empiriques ont été proposées pour calculer le coefficient de stabilité d'une digue (loi de Hudson, loi de Van der Meer, ... ; CAMINADE, 1995), des formules qui s'articulent en général sur quelques données comme la pente du talus, le poids des enrochements et les densités de fluide et de solide, mais elles ne prennent en compte ni la forme ni la juxtaposition des blocs, ni aucun des phénomènes cités ci-dessus, malgré cela, ces formules sont encore utilisées actuellement par plusieurs constructeurs de digues, dont le ministère de l'équipement.

Dans le présent travail, on se propose d'étudier numériquement la stabilité de ces structures sous sollicitations hydrodynamiques, en tenant compte de la forme des blocs, de leur dispositions, des forces de contact entre blocs, de la nature de l'écoulement du fluide (transitoire, périodiques, non linéaire) et de l'effet rétroactif de l'écoulement du fluide sur le système de blocs. Un couplage d'interaction fluide-structure est alors mis en œuvre pour simuler les mouvements des structures (blocs) sous les effets de la houle et des courants. Dans ce couplage, la structure est supposée formée d'éléments discrets (blocs) susceptibles de se déformer. Ainsi, la modélisation des mouvements des structures est effectuée par un modèle numérique d'éléments discrets basé sur la méthode de l'Analyse de Déformations Discontinues (DDA), tout en adoptant l'algorithme du 'Lagrangien Augmenté' pour la prise en compte des liaisons des contacts. Pour la partie fluide, un modèle numérique en éléments finis, basé sur les équations de Navier-Stokes, est utilisé pour déterminer les sollicitations hydrodynamiques qui agissent sur les blocs. La procédure de couplage est testée et validée sur des cas-test académiques et réels.

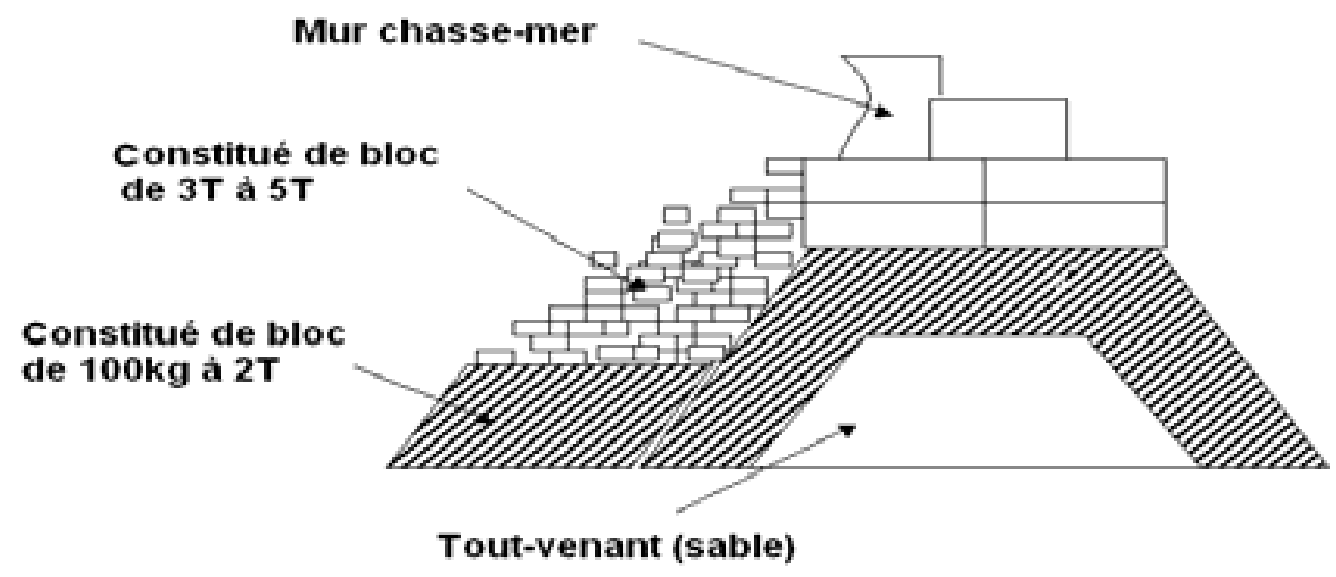

Figure 1. Présentation schématique de différents constituants d'une digue. 


\section{Modélisation du milieu solide}

\subsection{Formulation mathématique de l'Analyse des Déformations Discontinues (DDA)}

La DDA est une méthode numérique novatrice, proposée initialement par SHI (1988). Elle fait partie de la famille DEM utilisée généralement pour modéliser les milieux discrets. Son principe repose sur la minimisation de l'énergie potentielle, pour modéliser les déplacements d'un milieu solide discontinu et déformable, en tenant compte du contact aux différentes discontinuités de ce milieu.

Contrairement aux méthodes classiques de résolution numérique (FEM, DEM), la méthode DDA jouit de la capacité de gérer des discontinuités, aussi bien pour des surfaces de contact concaves ou convexes, et sans que ces surfaces soient précisées au préalable (KE \& BRAY, 1995). Cela veut dire que l'algorithme de contact de la méthode DDA procède à une auto-identification des zones de discontinuité.

L'énergie potentielle totale du système est définie par l'équation suivante :

$\Pi_{i}=f^{T} d_{B}+\frac{1}{2} \int \varepsilon^{T} \sigma d A$.

où $f^{T}$ est le vecteur force incluant les forces de contact, $d_{B}$ le déplacement, $\varepsilon$ la déformation, et $\sigma$ la contrainte.

Dans le cas bidimensionnel, le déplacement $(u, v)$ de chaque point $(x, y)$ du bloc $i$, est donné par :

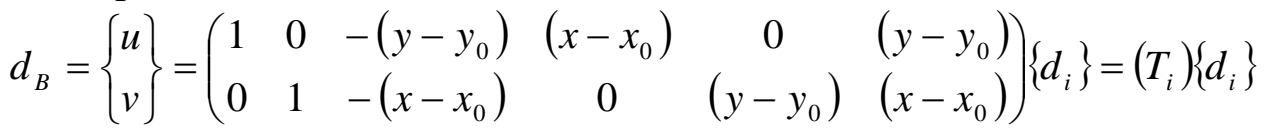

où $d_{i}$ désigne le vecteur des variables d'un bloc $i$, ce vecteur contient les translations $u_{0}, v_{0}$, la rotation de ce bloc autour de son centre de gravité $y_{0}$, et les déformations linéaires $\varepsilon_{x}, \varepsilon_{y}$ et $\varepsilon_{x y}$ respectivement :

$d_{i}{ }^{T}=\left\{u_{0}, v_{0}, y_{0}, \varepsilon_{x}, \varepsilon_{y}, \varepsilon_{x y}\right\}$

En minimisant l'énergie potentielle totale du système (1), on obtient les équations d'équilibre (4), à partir desquelles on déduit les déplacements de chaque bloc. Ces équations vérifient :

$\frac{\partial \Pi_{i}}{\partial d_{i}}=0, \quad$ où $i=1,6$

Après un calcul intermédiaire on aboutit au système diagonal suivant.

$K_{i i} D_{i}=F_{i}$

\subsection{Procédure de contact}

Lors de la procédure de contact entre blocs supposés solides et rigides, l'équilibre dynamique doit être assuré à chaque pas de temps $\Delta t$, et la pénétration entre ces blocs n'est pas autorisée (CHIHSEN, 1995). Toutefois, dans les présentes applications, pour des raisons de stabilité numérique, et afin de respecter cette contrainte de non 
pénétration, on introduit la méthode du "Lagrangien augmenté" (ROUAINIA et al., 2001) sous la forme suivante :

$\Pi_{S}=\lambda_{k}^{*} d+\frac{1}{2} p \cdot d^{2}$

où $\Pi_{S}$ représente l'énergie potentielle liée à la force de contact entre blocs, $d$ la distance ou gap de pénétration, $p$ le paramètre de pénalité, $\lambda_{k}{ }^{*}$ une force de contact inconnue qui caractérise la réaction qui s'oppose à la pénétration entre blocs. Le calcul de cette force est assuré par l'algorithme itératif suivant :

$\lambda_{k+1}^{*}=\lambda_{k}^{*}+p d$

$\lambda_{k}^{*}$ est la solution à l'itération $k, \lambda_{k+1}^{*}$ est la solution à l'itération $k+1$.

La prise en compte du contact entre blocs conduit au système suivant :

$K_{K} D_{K}=F_{K}$

Ensuite, en minimisant l'équation (6) et en assemblant les systèmes (5) et (7) on obtient le système global $(8)$ :

$\left(\begin{array}{cccc}k_{11} & k_{12} & \ldots & k_{1 n} \\ k_{21} & k_{22} & \ldots & k_{2 n} \\ \ldots & \ldots & \ldots & \ldots \\ k_{n 1} & k_{n 2} & \ldots & k_{n n}\end{array}\right)\left(\begin{array}{c}d_{1} \\ d_{2} \\ \ldots \\ d_{n}\end{array}\right)=\left(\begin{array}{c}f_{1} \\ f_{2} \\ \ldots \\ f_{n}\end{array}\right)$

où $f_{i}$ est le vecteur des forces extérieures appliquées sur le bloc $i$, qui inclut les forces de contact, $d_{i}$ est le vecteur de variables associées au bloc $i$, les termes de la diagonale $\left(k_{i i}, i=j\right)$ correspondent à la rigidité du matériau du bloc, les autres termes $\left(k_{i j}, i \neq j\right)$ représentent la rigidité associé au contact entre le bloc $i$ et le bloc $j$.

\section{Modélisation du milieu fluide : formulation mathématique}

On considère le mouvement d'un fluide, visqueux et incompressible, dans un repère cartésien Oxz. En notant $\Omega$ le domaine occupé par le fluide, l'écoulement est gouverné par les équations de conservation de la quantité de mouvement et de conservation de la masse. Ces équations sont les suivantes :

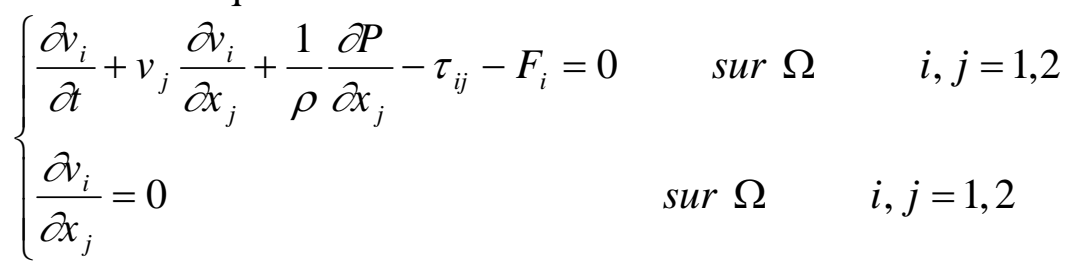

$\mathrm{v}_{\mathrm{i}}=(\mathrm{U}, \mathrm{W})$ est la vitesse suivant la direction $\mathrm{i}$, $\mathrm{P}$ est la pression dynamique, $F_{i}$ les composantes des forces extérieures et $\tau_{i j}$ les composantes du tenseur des contraintes visqueuses.

Ces équations ont été résolues en utilisant la méthode des éléments finis (DHATT \& TOUZOT, 1984). A ce système d'équations (9) sont associées les conditions aux limites suivantes : 


\section{XI $I^{\text {èmes }}$ Journées Nationales Génie Côtier - Génie Civil}

Les Sables d'Olonne, 22-25 juin 2010

- $\mathrm{U}=\mathrm{U}_{0}$, constante ou périodique à l'entrée du domaine (entrée amont gauche)

- $\mathrm{U}=\mathrm{W}=0$, au niveau des frontières solides,

- $\mathrm{p}=\mathrm{p}_{0}$, au niveau à la sortie du domaine.

\section{Procédure de couplage}

Le couplage entre les différents modules est effectué à chaque pas de temps. La pression obtenue par le module numérique fluide est utilisée comme sollicitation concentrée externe pour le module numérique solide. Un calcul itératif de ce dernier fournira les champs des déplacements des blocs de la digue. Après chaque étape, le calcul des variables fluides nécessitera un remaillage du domaine.

Dans les présentes investigations, les hypothèses suivantes sont utilisées :

- l'écoulement est supposé confiné (absence de surface libre),

- les blocs sont des polygones de forme quelconques,

- les sollicitations hydrodynamiques sont dues au courant seul (pas de houle),

- la digue est immergée.

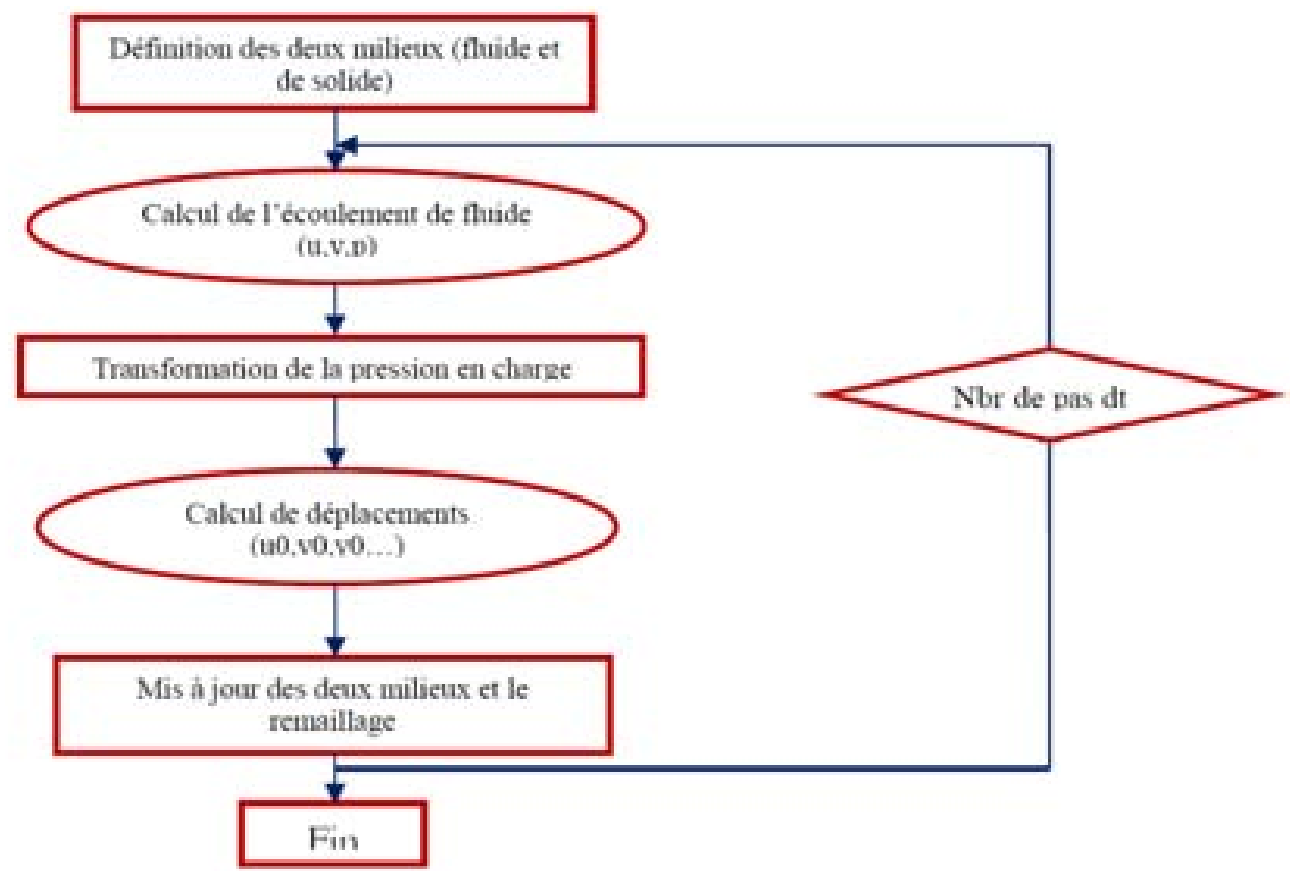

Figure. 2. Organigramme du couplage entre les modèles couplés.

\section{Applications et résultats numériques : cas test}

Ce cas test concerne la simulation de la stabilité d'un empilement formé de 6 billes. Le temps du calcul est de 0.3125 secondes, la vitesse à l'entrée du canal est de $0.3 \mathrm{~m} / \mathrm{s}$, la pression à la sortie est égale à la pression atmosphérique ( 1 bar), la vitesse au niveau des parois est nulle et enfin la masse volumique du solide est $2500 \mathrm{~kg} / \mathrm{m}^{3}$. 


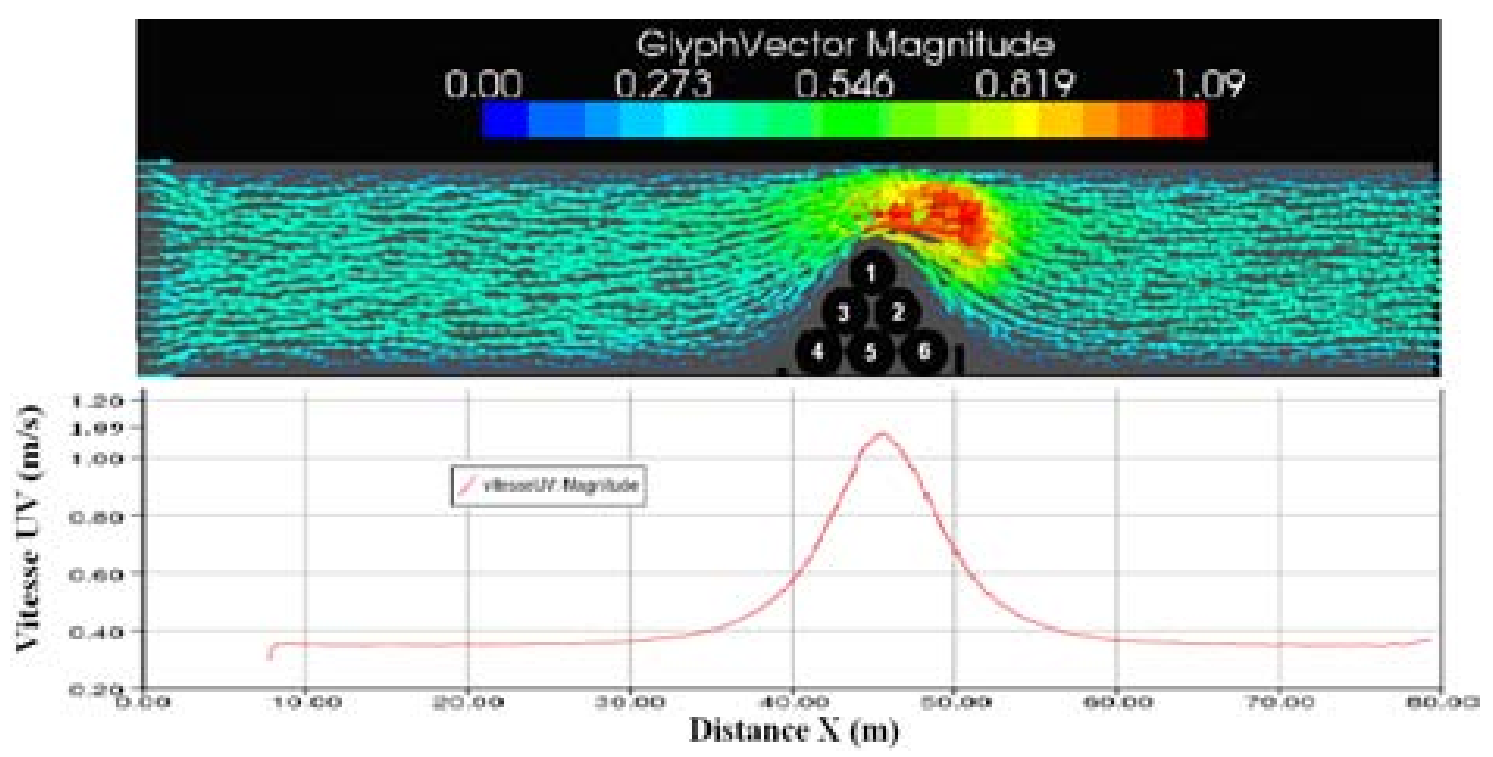

Figure. 3. Vitesse du fluide donnée par le code.

Les figures 3 et 4 montrent la vitesse en amont et en aval de l'empilement. En comparant les deux résultats, expérimental et numérique, on trouve que les deux vitesses sont très proches sauf qu'au niveau de l'empilement la vitesse monte jusqu'à $1 \mathrm{~m} / \mathrm{s}$, ce qui peut être expliqué par la nature de l'écoulement qui est confiné, mais aussi par les pores qui existent entre les billes en réalité mais qui ne peuvent pas être pris en considération tant qu'on travaille en 2D.
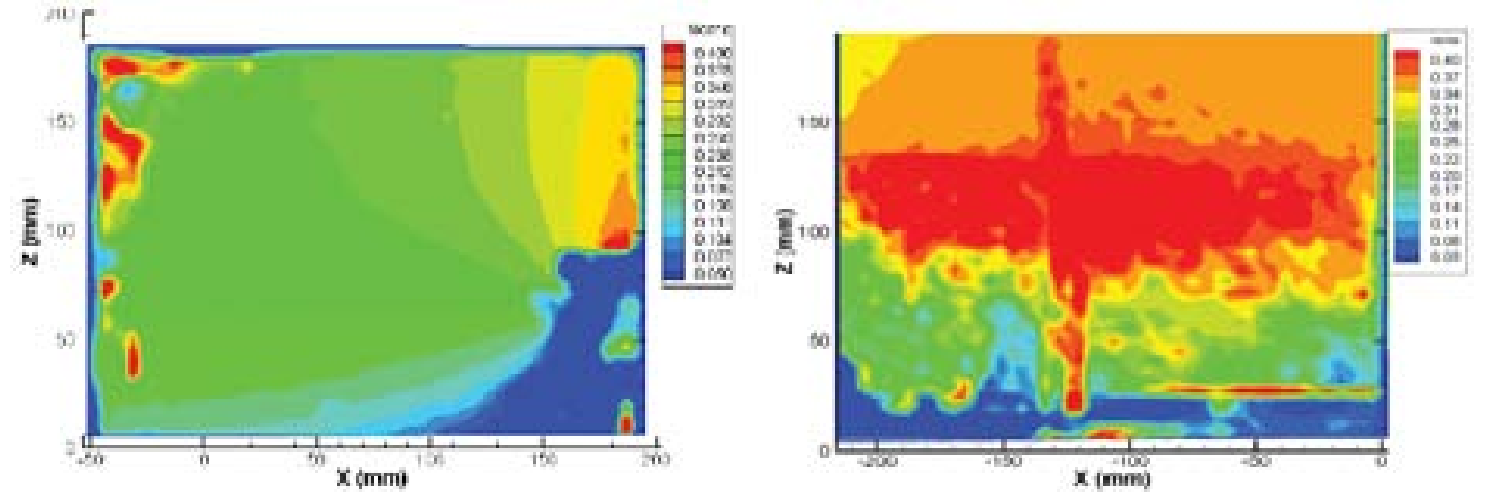

Figure. 4 - Vitesse expérimentale du fluide en amont et en aval de l'empilement.

En analysant les mouvements des billes identifiés par les chiffres de 1 à 3 (figure 5), on s'aperçoit que la porosité et le fait que le milieu est confiné jouent un rôle important sur le mouvement des billes, on remarque que dans les résultats numériques la bille numéro 1 bouge au premier avant les billes 2 et 3 ce qui n'est pas le cas dans la réalité. 


\section{XI $I^{\text {èmes }}$ Journées Nationales Génie Côtier - Génie Civil}

Les Sables d'Olonne, 22-25 juin 2010
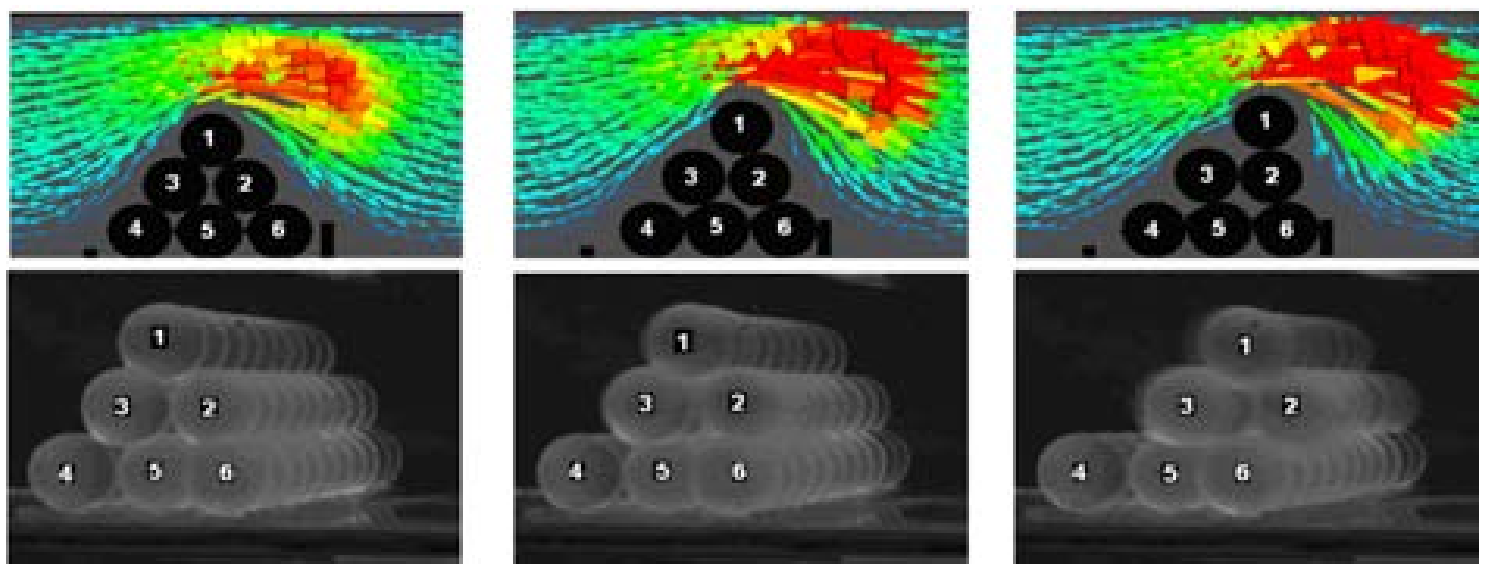

Figure. 5. Evolution temporelle de billes.

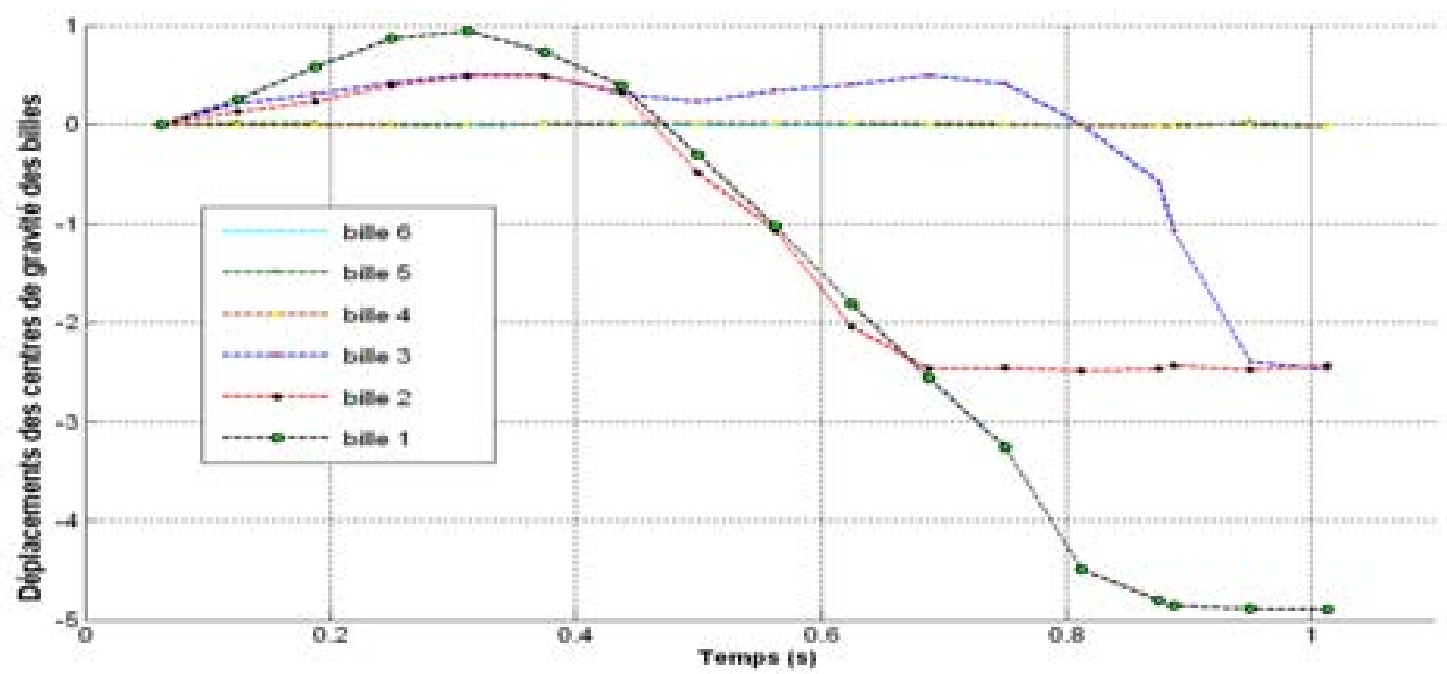

Figure. 6. Déplacement du centre de gravité des billes suivant $Y$.

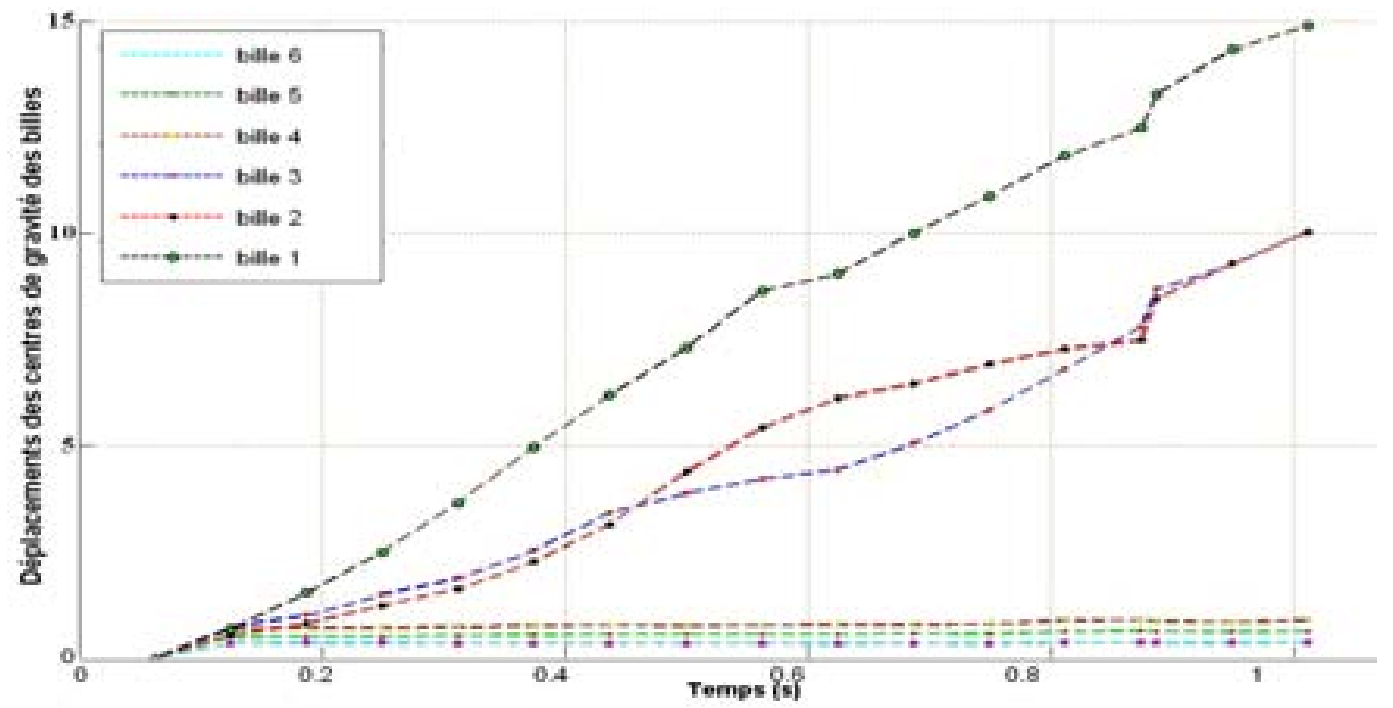

Figure. 7. Déplacement du centre de gravité des billes suivant $X$. 
On constate aussi que les 3 billes supérieures subissent des mouvements relativement brusques et rapides suivant $\mathrm{X}$ et ça, pendant toute la période de la simulation, par contre, suivant $\mathrm{Y}$ on remarque la même chose avec des déplacements plus faibles, et après un certain temps, les billes atteignent un état stable et ne bougent plus verticalement. Enfin malgré l'écart existant entre les mesures trouvées expérimentalement et numériquement, les deux résultats restent comparables et proche.

\section{Conclusion}

Un couplage entre deux modèles numériques (fluide et solide) est mis en œuvre pour étudier la stabilité des digues en enrochements. Il s'agit d'un couplage fluide structure où la structure est formée d'éléments discrets (blocs) susceptibles de se déformer. En utilisant la méthode DDA, comme alternative aux méthodes d'éléments finis classiques, nous avons pu introduire le contact entre les blocs et gérer les interactions blocs-fluide et blocs-blocs en optimisant le temps de calcul et la taille de la mémoire de stockage.

Notons que les effets de la houle et du milieu poreux n'ont pas été prise en compte. L'introduction de ces effets dans notre code de couplage constituera la phase suivante de ce travail.

\section{Références bibliographiques}

CAMINADE D. (1995). Ouvrages de protection contre la houle. Réf C 4631, Technique de l'Ingénieur.

CHIHSEN T. (1995). Extensions to the discontinuous deformation analysis for jointed rock masses and other blocky systems. Thèse de l'Université du Colorado, USA.

DHATT G., TOUZOT G. (1984). Une présentation de la méthode des éléments finis. Ed. Maloine, Paris.

KE T.C., BRAY J.D. (1995). Modeling of particulate media using discontinuous deformation analysis. Journal of Engineering Mechanics, Vol. 121, Issue 11, pp 1234-1243. doi:10.1061/(ASCE)0733-9399(1995)121:11(1234)

ROUAINIA M., PEARCE C., BICANIC N. (2001). HYDRO-DDA modelling of fractured mudrock seals. in: N.Bicanic (eds.) Proc. Int. Conf. on Analysis of Discontinuous Deformation: ICADD-4, Glasgow, Scotland, U.K, 6-8 June 2001.

SHI G.H. (1988). Discontinuous deformation analysis, a new numerical model for statics and dynamics of block systems. Thèse de l'Université de Californie, Berkeley, USA. 